\title{
Complete dynamic model of the Twin Rotor MIMO System (TRMS) with experimental validation
}

\author{
Azamat Tastemirov ${ }^{\mathrm{a}}$, Andrea Lecchini-Visintini ${ }^{\mathrm{a}, *}$, Rafael M. Morales ${ }^{\mathrm{a}}$ \\ ${ }^{a}$ Department of Engineering, University of Leicester, UK
}

\begin{abstract}
In this paper we develop a complete dynamic model of the Twin Rotor MIMO System (TRMS) using the Euler-Lagrange method. Our model improves upon the model provided by the manufacturer in the user manual and upon previous models of the TRMS which can be found in the literature. The complete procedure for the model parameters' estimation and validation is illustrated.

Keywords: Twin Rotor MIMO System (TRMS), dynamic models, Euler-Lagrange method
\end{abstract}

\section{Introduction}

The Twin Rotor MIMO Systems (TRMS) - shown in Figure 1 - consists of a beam rotating freely in the vertical plane (pitch) about the end of a pivoted beam, which in turn rotates in the horizontal plane (yaw) about a fixed point. The beam is damped by a perpendicular counterbalance beam rigidly fixed in its centre. The beam is driven by two mutually perpendicular propellers located at its ends and driven by DC motors. The pitch angles of the propeller blades are fixed, thus the propulsive force is governed by the propeller speed of rotation. The TRMS is characterised by a highly non-linear and cross-coupled dynamics, and is a challenging system to control. For this reason, it has been the subject of many works over the last decade see e.g. $[1,2,3,4,5]$ and references therein.

The simplified Newtonian models provided by the manufacturer in $[6,7]$ do not capture the system's dynamics precisely. An extensive attempt to overcome the limitations of these models was made in [3], where an updated version of the Newtonian model provided by the manufacturer and a new model, derived following an Euler-Lagrange approach, were presented. The Newtonian model model of [3] was subsequently updated and improved in [4], where it was used as the base model for LPV modeling and identification of the TRMS dynamics. The application of the Newtonian approach to obtain the equations of the complex TRMS dynamics requires taking into account a variety of fictitious forces, the parameters of which are not readily available, while the Euler-Lagrange approach provides a more natural way to obtain dynamical equations. The

${ }^{*}$ Corresponding author. Email: alv1@leicester.ac.uk 


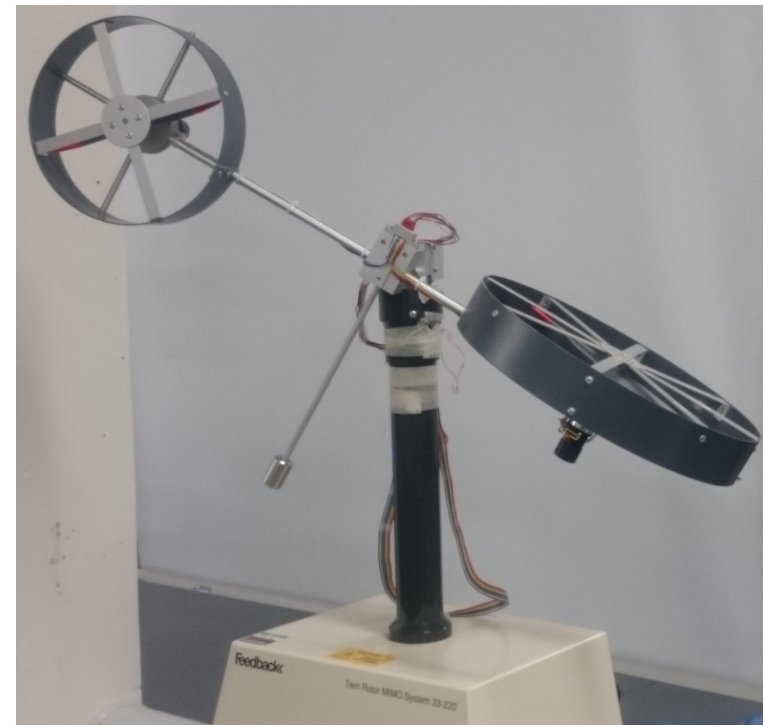

Figure 1: The Twin Rotor MIMO System (Feedback Instruments)

application of the Euler-Lagrange approach to the equations of motion of the TRMS was also reported in [2] but without disclosing the derivation, with final equations presented in their most general form, and without providing numerical values of parameters.

In this contribution, we obtain a complete dynamic model of the TRMS explicitly derived through application of the Euler-Lagrange approach. We provide the reader with numerical values of all parameters, which have been identified using experimental data, and we illustrate the performance of the model with a detailed experimental validation. In our work, we adopt the modelling methodology presented in [8], where it was applied to a similar system called Toycopter. This approach is characterised by the use of body-fixed frames of reference to express all vector quantities of the translating parts, a choice which significantly simplifies the calculation of the kinetic energies. The main structural difference between the Toycopter and the TRMS is the presence of a pivoted beam, of a counterbalance beam, and of a flat cable, in the latter, which result in more complex derivations and final dynamical equations. In a comparison with earlier models we show that our model overcomes some inaccuracies of the models provided in [3] and [4]. This is an important aspect of the contribution since the model of [3] and [4] continues to be the model of choice in recent works focused on the control of the TRMS, see e.g. [5]. In addition, we show that we recover the general equations of the model of [2] but with an explicit expression for each term. Hence, the TMRS model provided in this work can be readily used as a test-bench to develop new control strategies for the TRMS.

A preliminary version of this paper was presented at the 39th European Rotorcraft Forum [9]. 


\section{Twin Rotor dynamics}

We will first obtain the equations describing the DC motors and then the equations of motion of the mechanical parts.

\subsection{DC motors}

The main and tail DC motors are assumed to be identical, therefore, the same equations will be used for each motor by adding the subscript " $\mathrm{m}$ " or " $\mathrm{t}$ ".

The voltage of the DC motor, denoted as $v$, is set in Simulink through the control signal $u$ with range $[-2.5,2.5]$. This channel is assumed to have

a constant gain, i.e. $v=k_{u} u$. The DC motor itself is described by a simple first-order differential equation

$$
L_{m} \frac{d i}{d t}=v-k_{v} \omega-R i
$$

where: $i$ is the motor current, $L_{m}$ is the motor inductance, $R$ is the motor resistance, $k_{v}$ is the motor back EMF constant, $\omega$ is the motor angular velocity. Using the parameters' values (obtained in the following sections) we can compare the numerical values of the electrical and mechanical time constants of, for example, the main rotor

$$
c_{e}=\frac{L_{m m}}{R_{m}}=1.075 \times 10^{-4} \mathrm{~s} \quad c_{m}=\frac{I_{m 1} R_{m}}{k_{t m} k_{v m}}=3.38 \mathrm{~s}
$$

and note that the dynamics of the motor's current can be neglected, resulting in the following algebraic equation for the DC motor circuit:

$$
R i=v-k_{v} \omega .
$$

Each DC motor generates an electromechanical torque which is assumed be proportional to the current.

\subsection{Rigid body}

We will follow a standard Euler-Lagrange approach [10]. We will adopt generalised coordinates

$$
\mathbf{q}=\left[\begin{array}{llll}
\psi & \phi & \rho_{m} & \rho_{t}
\end{array}\right]^{T}
$$

where $\psi$ denote the pitch angle, $\phi$ the yaw angle, $\rho_{m}$ and $\rho_{t}$ the angles of the main and tail rotors as shown in Figure 2. We will use $T(\mathbf{q}, \dot{\mathbf{q}}, t)$ and $U(\mathbf{q}, t)$ to denote kinetic and potential energy respectively, and $F_{q_{i}}$ to denote the generalised force for each coordinate. Then using the the Lagrangian equation $L(\mathbf{q}, \dot{\mathbf{q}}, t)=T(\mathbf{q}, \dot{\mathbf{q}}, t)-U(\mathbf{q}, t)$ we obtain the equations of motion from

$$
\frac{d}{d t}\left(\frac{\partial L}{\partial \dot{q}_{i}}\right)-\frac{\partial L}{\partial q_{i}}=F_{q_{i}}
$$




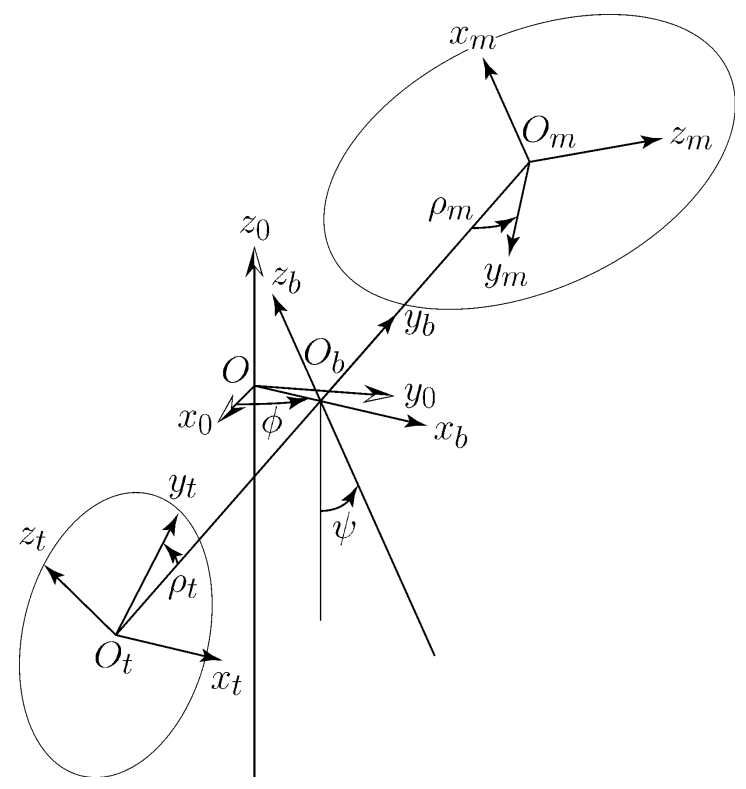

Figure 2: TRMS notation

\section{Kinetic energy}

Consider a rigid body, performing an arbitrary motion in three dimensional space. Let $M$ denote the mass of the body. Let $A$ denote an arbitrary point fixed in the body, and $\mathbf{I}_{\mathbf{A}}$ the inertia matrix with respect to the point $A$. Furthermore, let $\mathbf{v}_{\mathbf{A}}$ denote the instantaneous linear velocity vector of $A, \boldsymbol{\Omega}$ the instantaneous angular velocity vector, which is invariant with respect to the choice of $A$, and $\mathbf{r}_{\mathrm{AG}}$ the vector between the centre of mass $G$ and $A$. The kinetic energy of the rigid body can be obtained using the following general formula [11, eq. (5.2)]:

$$
T=\frac{1}{2} M \mathbf{v}_{\mathbf{A}}^{\mathbf{T}} \mathbf{v}_{\mathbf{A}}+M \mathbf{v}_{\mathbf{A}}^{\mathbf{T}}\left(\boldsymbol{\Omega} \times \mathbf{r}_{\mathbf{A G}}\right)+\frac{1}{2} \boldsymbol{\Omega}^{\mathbf{T}} \mathbf{I}_{\mathbf{A}} \boldsymbol{\Omega} .
$$

In order to calculate the total kinetic energy the TRMS will be considered consisting of four separate rigid bodies, namely: $a$ ) main rotor (main propeller and associated rotating parts); $b$ ) tail rotor (tail propeller and associated rotating parts); c) body, comprising the beam, rotors shields and counterbalance beam with weight; and $d$ ) pivoted beam. Each rotor is formed by the corresponding propeller and DC motor rotor. In order to simplify the calculation of the kinetic energy, all vector quantities associated with each body are expressed in its body-fixed frame, i.e. reference frame attached to the body.

- Let the body-fixed frame $\left(x_{m}, y_{m}, z_{m}\right)$ of the main rotor be attached to its centre of mass $O_{m}$ so that its $x_{m}$-axis coincides with the axis of rotation of the propeller and is directed upwards, and $y_{m}$-axis coincides with the propeller blade (Figure 2). The main rotor angular velocity is determined as the vector 
sum of three angular velocities of rotation along $\rho_{m}, \psi$ and $\phi$ angles (Figure 3):

$$
\boldsymbol{\Omega}_{\mathbf{m}}=\left[\begin{array}{c}
\dot{\rho}_{m}+\dot{\phi} \cos \psi \\
\dot{\psi} \sin \rho_{m}-\dot{\phi} \cos \rho_{m} \sin \psi \\
\dot{\psi} \cos \rho_{m}+\dot{\phi} \sin \rho_{m} \sin \psi
\end{array}\right] .
$$

Let $l_{m}$ denote the distance between the points $O_{b}$ and $O_{m}$ and $d_{m}$ the distance between the points $O$ and $O_{m}$. Translation of the point $O_{m}$ is due to the rotation of the beam along the $\psi$ and $\phi$ angles. The instantaneous linear velocity vector of the point $O_{m}$ with respect to the body-fixed frame is (Figure 4):

$$
\begin{aligned}
\mathbf{v}_{\mathbf{m}} & =\left[\begin{array}{c}
l_{m} \dot{\psi}-d_{m} \dot{\phi} \sin \alpha \cos \beta \sin \psi \\
-d_{m} \dot{\phi}(\sin \alpha \cos \beta \cos \psi \cos \rho+\sin \alpha \sin \beta \sin \rho) \\
-d_{m} \dot{\phi}(-\sin \alpha \cos \beta \cos \psi \sin \rho+\sin \alpha \sin \beta \cos \rho)
\end{array}\right] \\
= & {\left[\begin{array}{c}
l_{m} \dot{\psi}-d_{m} \dot{\phi} \sin \theta \sin \psi \\
-d_{m} \dot{\phi} \cos \psi \sin \left(\rho_{m}+\theta\right) \\
-d_{m} \dot{\phi} \cos \psi \cos \left(\rho_{m}+\theta\right)
\end{array}\right] }
\end{aligned}
$$

where $\theta$ is a fixed angle determined as $\arccos \left(l_{m} / d_{m}\right)$. In Figure 4 , the point $P_{m}$ is the projection of $O_{m}$ onto the plane defined by the $x_{0}$ and $y_{0}$ axes. Note that, for the sake of clarity of representation, the contribution of $\dot{\phi}$ is depicted for the case $\rho_{m}=0$. Equation (8) is then obtained by introducing a rotation around the $x_{m}$ axis. The expressions in (9) are finally obtained from the simple geometric relations $\sin \alpha \cos \beta=\sin \theta$ and $\sin \alpha \sin \beta=\cos \psi \cos \theta$. Under some geometric assumptions, which will introduced in Section 3, the tensor of inertia of the main rotor is diagonal:

$$
\mathbf{I}_{\mathbf{m}}=\left[\begin{array}{ccc}
I_{m 1} & 0 & 0 \\
0 & I_{m 2} & 0 \\
0 & 0 & I_{m 3}
\end{array}\right] .
$$

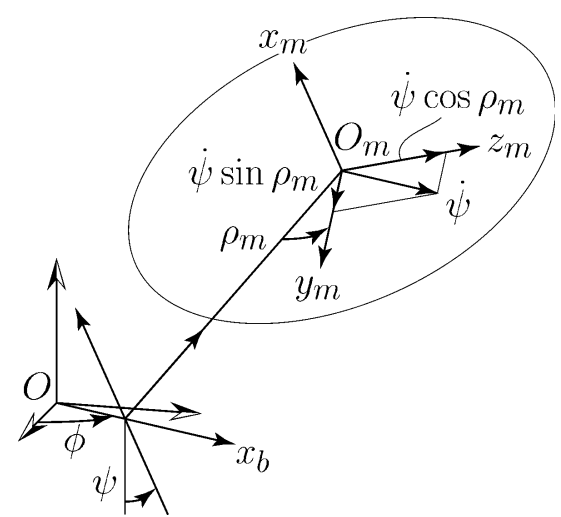

(a) Contribution of $\dot{\psi}$

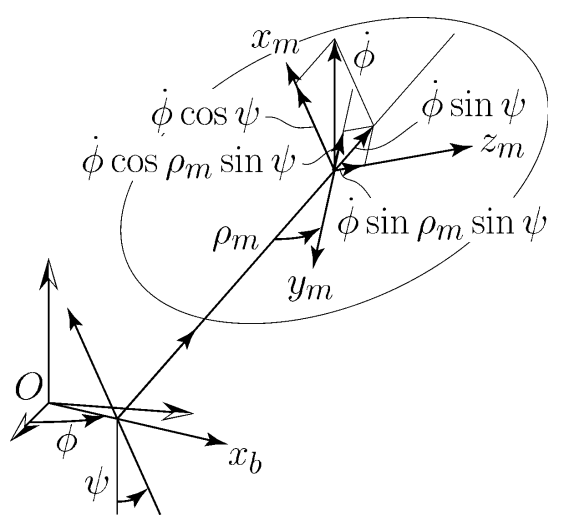

(b) Contribution of $\dot{\phi}$

Figure 3: Calculation of the main rotor angular velocity $\left(\boldsymbol{\Omega}_{\mathbf{m}}\right)$ 


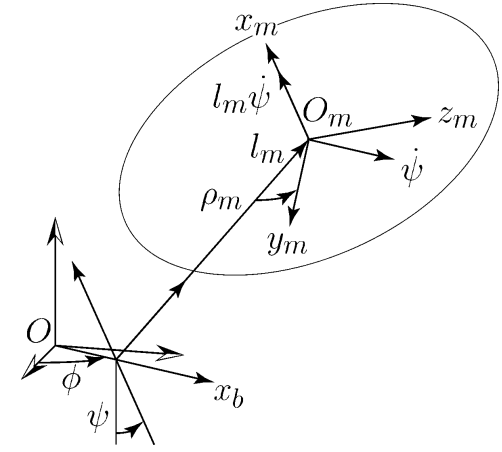

(a) Contribution of $\dot{\psi}$

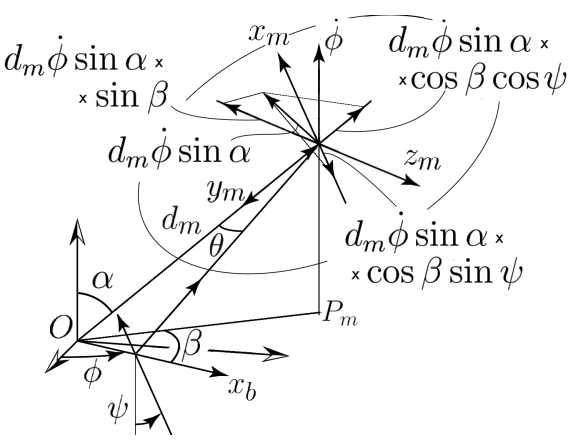

(b) Contribution of $\dot{\phi}$ when $\rho_{m}=0$

Figure 4: Calculation of the main rotor linear velocity $\left(\mathbf{v}_{\mathbf{m}}\right)$

Due to the choice of the body-fixed frame, the position vector (corresponding to $\mathbf{r}_{\mathbf{A G}}$ in $\left.(6)\right)$ vanishes.

- Let $h$ denote the distance between the points $O_{b}$ and $O$. Applying the same approach to the tail rotor we obtain:

$$
\begin{gathered}
\boldsymbol{\Omega}_{\mathbf{t}}=\left[\begin{array}{lll}
\dot{\psi}+\dot{\rho}_{t} & \dot{\phi} \sin \left(\psi+\rho_{t}\right) & \dot{\phi} \cos \left(\psi+\rho_{t}\right)
\end{array}\right]^{T} \\
\mathbf{v}_{\mathbf{t}}=\left[\begin{array}{c}
l_{t} \dot{\phi} \cos \psi \\
-l_{t} \dot{\psi} \sin \rho_{t}+h \dot{\phi} \cos \left(\psi+\rho_{t}\right) \\
-l_{t} \dot{\psi} \cos \rho_{t}-h \dot{\phi} \sin \left(\psi+\rho_{t}\right)
\end{array}\right] \\
\mathbf{I}_{\mathbf{t}}=\left[\begin{array}{ccc}
I_{t 1} & 0 & 0 \\
0 & I_{t 2} & 0 \\
0 & 0 & I_{t 3}
\end{array}\right]
\end{gathered}
$$

- Let the body-fixed frame $\left(x_{b}, y_{b}, z_{b}\right)$ of the TRMS body be attached to point $O_{b}$ (Figure 2). The angular velocity vector of the TRMS body with respect to the body-fixed frame is

$$
\boldsymbol{\Omega}_{\mathbf{b}}=\left[\begin{array}{lll}
\dot{\psi} & \dot{\phi} \sin \psi & \dot{\phi} \cos \psi
\end{array}\right]^{T}
$$

and the linear velocity vector of the point $O_{b}$ due to rotation of the TRMS body along $\phi$ angle is

$$
\mathbf{v}_{\mathbf{b}}=\left[\begin{array}{lll}
0 & h \dot{\phi} \cos \psi & -h \dot{\phi} \sin \psi
\end{array}\right]^{T} .
$$

Let $G_{b}$ denote the centre of mass of the TRMS body. Due to symmetry $G_{b}$ lies somewhere in the plane formed by the main and counterbalance beams. The corresponding position vector, to be used in equation (6), is:

$$
\mathbf{r}_{\text {ObGb }}=\left[\begin{array}{lll}
0 & y_{G b} & z_{G b}
\end{array}\right]^{T} .
$$


It can be easily demonstrated that the tensor of inertia of the TRMS body is diagonal of form:

$$
\mathbf{I}_{\mathbf{b}}=\left[\begin{array}{ccc}
I_{b 1} & 0 & 0 \\
0 & I_{b 2} & 0 \\
0 & 0 & I_{b 3}
\end{array}\right] .
$$

We are now in the position to calculate the kinetic energy of the rigid body. Let $M_{m}, M_{t}$ and $M_{b}$ denote the masses of the main propeller and associated rotating parts, of the tail propeller and associated rotating parts, and of the TRMS body respectively. Expanding equation (6) for each component using the equations obtained above yields the following equations for the kinetic energies:

$$
\begin{aligned}
T_{m} & =\frac{1}{2} I_{m 1} \dot{\rho}_{m}^{2}+I_{m 1} \dot{\rho}_{m} \dot{\phi} \cos \psi+\frac{1}{2} I_{m 1} \dot{\phi}^{2} \cos ^{2} \psi \\
& +\frac{1}{2}\left(I_{m 2} \sin ^{2} \rho_{m}+I_{m 3} \cos ^{2} \rho_{m}\right) \dot{\psi}^{2}+\frac{1}{2}\left(I_{m 2} \cos ^{2} \rho_{m}+I_{m 3} \sin ^{2} \rho_{m}\right) \dot{\phi}^{2} \sin ^{2} \psi \\
& +\left(I_{m 3}-I_{m 2}\right) \sin \rho_{m} \cos \rho_{m} \dot{\phi} \dot{\psi} \sin \psi \\
& +\frac{1}{2} M_{m} l_{m}^{2} \dot{\psi}^{2}+\frac{1}{2} M_{m} h^{2} \dot{\phi}^{2} \sin ^{2} \psi+\frac{1}{2} M_{m} d_{m}^{2} \dot{\phi}^{2} \cos ^{2} \psi-M_{m} h l_{m} \dot{\phi} \dot{\psi} \sin \psi \\
T_{t} & =\frac{1}{2} I_{t 1} \dot{\psi}^{2}+I_{t 1} \dot{\rho}_{t} \dot{\psi}+\frac{1}{2} I_{t 1} \dot{\rho}_{t}^{2}+\frac{1}{2} I_{t 2} \dot{\phi}^{2} \sin ^{2}\left(\psi+\rho_{t}\right)+\frac{1}{2} I_{t 3} \dot{\phi}^{2} \cos ^{2}\left(\psi+\rho_{t}\right) \\
& +\frac{1}{2} M_{t} l_{t}^{2} \dot{\psi}^{2}+\frac{1}{2} M_{t} l_{t}^{2} \dot{\phi}^{2} \cos ^{2} \psi+\frac{1}{2} M_{t} h^{2} \dot{\phi}^{2}+M_{t} h l_{t} \dot{\phi} \dot{\psi} \sin \psi \\
T_{b} & =\frac{1}{2} I_{b 1} \dot{\psi}^{2}+\frac{1}{2} I_{b 2} \dot{\phi}^{2} \sin ^{2} \psi+\frac{1}{2} I_{b 3} \dot{\phi}^{2} \cos ^{2} \psi+\frac{1}{2} M_{b} \dot{\phi}^{2} h^{2} \\
& -M_{b} \dot{\phi} \dot{\psi} h\left(z_{G b} \cos \psi+y_{G b} \sin \psi\right) .
\end{aligned}
$$

Let us recall that in these expressions $h$ denotes the distance between the points $O_{b}$ and $O, d_{m}$ denotes the distance between the points $O$ and $O_{m}$, and $d_{t}$ denotes the distance between the points $O$ and $O_{t}$, and that $d_{m}^{2}=l_{m}^{2}+h^{2}$, $d_{t}^{2}=l_{t}^{2}+h^{2}$ and $h=d \sin (\theta)$. The kinetic energy of the pivoted beam is simply:

$$
T_{p}=\frac{1}{2} I_{p} \dot{\phi}^{2}
$$

\section{Potential energy}

In order to obtain the total potential energy we consider the aggregate body of mass $M_{a}$ consisting of those parts which have a variable potential energy: the main rotor, tail rotor and body. Let $G_{a}$ denote the centre of mass of the aggregate body. Due to symmetry, the position of $G_{a}$ with respect to the body-fixed frame can be defined by two coordinates $y_{G a}$ and $z_{G a}$. Expressing the vertical position of $\mathbf{G}_{\mathbf{a}}$ relatively to the stationary point $O$ in terms of $y_{G a}$ and $z_{G a}$ the potential

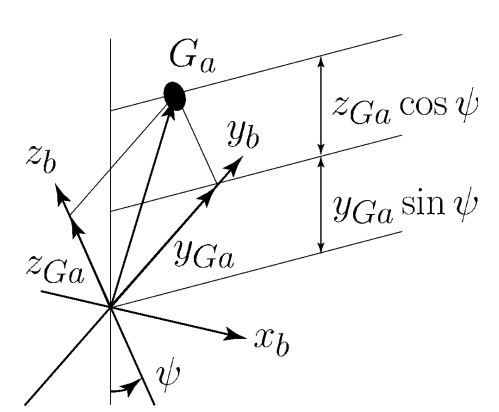

Figure 5: Vertical position of the centre of mass 
energy takes the form (Figure 5):

$$
V_{a}=M_{a} g\left(z_{G a} \cos \psi+y_{G a} \sin \psi\right) .
$$

\subsection{Lagrangian}

Expanding the Lagrangian $L=T_{m}+T_{t}+T_{b}+T_{p}-V_{a}$ and grouping the constants in the resulting expression yields

$$
\begin{aligned}
& L=\frac{1}{2}\left[I_{p}+\left(M_{m}+M_{t}+M_{b}\right) h^{2}\right] \dot{\phi}^{2}+\frac{1}{2}\left(I_{m 1}+I_{b 3}+M_{m} l_{m}^{2}+M_{t} l_{t}^{2}\right) \dot{\phi}^{2} \cos ^{2} \psi \\
& +\frac{1}{2} I_{b 2} \dot{\phi}^{2} \sin ^{2} \psi+\frac{1}{2} I_{m 2} \dot{\phi}^{2} \sin ^{2} \psi \cos ^{2} \rho_{m}+\frac{1}{2} I_{m 3} \dot{\phi}^{2} \sin ^{2} \psi \sin ^{2} \rho_{m} \\
& +\frac{1}{2} I_{t 2} \dot{\phi}^{2} \sin ^{2}\left(\psi+\rho_{t}\right)+\frac{1}{2} I_{t 3} \dot{\phi}^{2} \cos ^{2}\left(\psi+\rho_{t}\right)+\frac{1}{2}\left(I_{b 1}+I_{t 1}+M_{m} l_{m}^{2}+M_{t} l_{t}^{2}\right) \dot{\psi}^{2} \\
& +\frac{1}{2} I_{m 2} \dot{\psi}^{2} \sin ^{2} \rho_{m}+\frac{1}{2} I_{m 3} \dot{\psi}^{2} \cos ^{2} \rho_{m}+\frac{1}{2} I_{m 1} \dot{\rho}_{m}^{2}+\frac{1}{2} I_{t 1} \dot{\rho}_{t}^{2} \\
& +\left(M_{t} l_{t}-M_{m} l_{m}\right) h \dot{\phi} \dot{\psi} \sin \psi+\left(I_{m 3}-I_{m 2}\right) \dot{\phi} \dot{\psi} \sin \psi \cos \rho_{m} \sin \rho_{m}+I_{m 1} \dot{\phi} \dot{\rho}_{m} \cos \psi \\
& +I_{t 1} \dot{\psi} \dot{\rho}_{t}-M_{a} g z_{G a} \cos \psi-M_{a} g y_{G a} \sin \psi-M_{b} h z_{G b} \dot{\phi} \dot{\psi} \cos \psi-M_{b} h y_{G b} \dot{\phi} \dot{\psi} \sin \psi
\end{aligned}
$$

\section{Generalized forces}

The following forces are external to the TRMS.

1. The aerodynamic forces created by the propellers:

Each rotating propeller generates a propulsive force (thrust) and a reaction couple due to air resistance on the blades of the propeller (drag), which are both proportional to the square of the angular speed, see e.g. [12]. Hence, the main propeller generates the thrust $C_{T m} \dot{\rho}_{m}\left|\dot{\rho}_{m}\right|$ and, correspondingly, the thrust torque $C_{T m} \dot{\rho}_{m}\left|\dot{\rho}_{m}\right| l_{m}$ acting along the $\psi$ angle, the reaction torque $-C_{R m} \dot{\rho}_{m}\left|\dot{\rho}_{m}\right|$ acting along the $\rho_{m}$ angle, and the reaction torque $-C_{R m} \dot{\rho}_{m}\left|\dot{\rho}_{m}\right| \cos \psi$ acting along the $\phi$ angle. Similarly, the tail propeller generates the thrust torque $C_{T t} \dot{\rho}_{t}\left|\dot{\rho}_{t}\right| l_{t} \cos \psi$ along the $\phi$ angle, the reaction torque $-C_{R t} \dot{\rho}_{t}\left|\dot{\rho}_{t}\right|$ along the $\rho_{m}$ angle, and the reaction torque $-C_{R t} \dot{\rho}_{t}\left|\dot{\rho}_{t}\right|$ along the $\psi$ angle. Note that the $C_{T}$ and $C_{R}$ coefficients have different units of measure.

2. The electromechanical forces generated by DC motors:

Each DC motor generates an electromechanical torque equal to $k_{t} i$, where $k_{t}$ and $i$ stand for motor torque constant and current respectively.

3. The viscous forces due to friction in ball bearings:

We assume a simplified friction model, often utilized in the control of mechanical systems, which takes into account only viscous and Coulomb friction, see e.g. [13]. Moreover, assuming high rotational speed of the rotors, the Coulomb friction term for the corresponding coordinates can be neglected. Thus, the magnitudes of friction torques for each coordinate are given by $-\left(f_{v \psi} \dot{\psi}+f_{c \psi} \operatorname{sgn} \dot{\psi}\right),-\left(f_{v \phi} \dot{\phi}+f_{c \phi} \operatorname{sgn} \dot{\phi}\right),-f_{v m} \dot{\rho}_{m},-f_{v t} \dot{\rho}_{t}$. 
4. The force created by the wires:

The wires connecting the electrical equipment located on the moving parts of the TRMS with the electronic board at the base of the setup determine a rest yaw angle $\phi_{0}$ and a torque acting along the $\phi$ angle which is taken as $-C_{c}\left(\phi-\phi_{0}\right)$.

Summing up all the forces mentioned above for each of generalised coordinates yields:

$$
\begin{aligned}
F_{\psi} & =C_{T m} \dot{\rho}_{m}\left|\dot{\rho}_{m}\right| l_{m}-C_{R t} \dot{\rho}_{t}\left|\dot{\rho}_{t}\right|-f_{v \psi} \dot{\psi}-f_{c \psi} \operatorname{sgn} \dot{\psi} \\
F_{\phi} & =C_{T t} \dot{\rho}_{r}\left|\dot{\rho}_{r}\right| l_{t} \cos \psi-C_{R m} \dot{\rho}_{m}\left|\dot{\rho}_{m}\right| \cos \psi-f_{v \phi} \dot{\phi}-f_{c \phi} \operatorname{sgn} \dot{\phi} \\
& -C_{c}\left(\phi-\phi_{0}\right) \\
F_{\rho_{m}} & =k_{t m} i_{m}-f_{v m} \dot{\rho}_{m}-C_{R m} \dot{\rho}_{m}\left|\dot{\rho}_{m}\right| \\
F_{\rho_{t}} & =k_{t t} i_{t}-f_{v t} \dot{\rho}_{t}-C_{R t} \dot{\rho}_{t}\left|\dot{\rho}_{t}\right|
\end{aligned}
$$

\section{Equations of motion}

The equations of motion are derived by developing equation (5) and (23) in terms of each generalised coordinate. Under the assumption of high rotational speed, i.e. that $\psi, \phi, \dot{\rho}_{m}, \dot{\rho}_{t}$ present slow variations over the period of each rotor's revolution, the cyclic terms depending on $\rho_{m}$ and $\rho_{t}$ appearing in the equations of motion can be replaced by their average, e.g. $\cos \rho_{m}$ and $\cos ^{2} \rho_{m}$ can be replaced by 0 and 0.5 respectively. Hence, the following equations are obtained:

$$
\begin{gathered}
I_{\psi} \ddot{\psi}-\left(H_{z} \cos \psi+H_{y} \sin \psi\right) \ddot{\phi}+I_{t 1} \dot{\omega}_{t}=-\frac{1}{2} I_{c} \dot{\phi}^{2} \sin (2 \psi)-I_{m 1} \omega_{m} \dot{\phi} \sin \psi \\
+G_{z} \sin \psi-G_{y} \cos \psi+C_{T m} \omega_{m}\left|\omega_{m}\right| l_{m}-C_{R t} \omega_{t}\left|\omega_{t}\right|-f_{v \psi} \dot{\psi}-f_{c \psi} \operatorname{sgn} \dot{\psi} \\
-\left(H_{z} \cos \psi+H_{y} \sin \psi\right) \ddot{\psi}+\left(I_{\phi}+I_{c} \cos ^{2} \psi\right) \ddot{\phi}+I_{m 1} \dot{\omega}_{m} \cos \psi= \\
-\left(H_{z} \sin (\psi)-H_{y} \cos (\psi)\right) \dot{\psi}^{2}+I_{c} \dot{\phi} \dot{\psi} \sin (2 \psi)+I_{m 1} \omega_{m} \dot{\psi} \sin \psi \\
+C_{T t} \omega_{t}\left|\omega_{t}\right| l_{t} \cos \psi-C_{R m} \omega_{m}\left|\omega_{m}\right| \cos \psi-f_{v \phi} \dot{\phi}-f_{c \phi} \operatorname{sgn} \dot{\phi}-C_{c}\left(\phi-\phi_{0}\right) \\
I_{m 1} \dot{\omega}_{m}+I_{m 1} \ddot{\phi} \cos (\psi)=I_{m 1} \dot{\phi} \dot{\psi} \sin \psi+k_{t m} i_{m}-f_{v m} \omega_{m}-C_{R m} \omega_{m}\left|\omega_{m}\right| \\
I_{t 1} \dot{\omega}_{t}+I_{t 1} \ddot{\psi}=k_{t t} i_{t}-f_{v t} \omega_{t}-C_{R t} \omega_{t}\left|\omega_{t}\right|
\end{gathered}
$$

where: $I_{\psi}=I_{b 1}+I_{t 1}+M_{m} l_{m}^{2}+M_{t} l_{t}^{2}+I_{m 23}, I_{\phi}=I_{p}+\left(M_{m}+M_{t}+M_{b}\right) h^{2}+$ $I_{b 2}+I_{m 23}+I_{t 23}, I_{c}=I_{m 1}+I_{b 3}+M_{m} l_{m}^{2}+M_{t} l_{t}^{2}-I_{b 2}-I_{m 23}, H_{z}=M_{b} h z_{G b}$, $H_{y}=M_{b} h y_{G b}+\left(M_{m} l_{m}-M_{t} l_{t}\right) h, G_{y}=M_{a} g y_{G a}, G_{z}=M_{a} g z_{G a}, I_{m 23}=$ $0.5\left(I_{m 2}+I_{m 3}\right), I_{t 23}=0.5\left(I_{t 2}+I_{t 3}\right)$ and $\omega_{m}=\dot{\rho}_{m}$ and $\omega_{t}=\dot{\rho}_{t}$. 


\subsection{Comparison with previously reported models}

We now compare the equations of motion (28)-(31) with the models which have appeared previously in the literature. To start with, we point out that for $h=0$ (i.e. the distance between the points $O_{b}$ and $O$ ) we recover the structure of the Toycoper [8]. Equations (28)-(31) reflect this fact. Indeed, for $h=0$, we recover all terms of the equations of motion in [8].

For $h>0$, we have the additional inertial term $\left(H_{z} \cos \psi+H_{y} \sin \psi\right) \ddot{\phi}$ in the pitch equation (28), and the additional inertial $\left(H_{z} \cos \psi+H_{y} \sin \psi\right) \ddot{\psi}$ and centrifugal $\left(H_{z} \sin (\psi)-H_{y} \cos (\psi)\right) \dot{\psi}^{2}$ terms in the yaw equation (29). Equations (28)-(29) are consistent with the equations of motion in general form given in [2], apart for inertial terms $I_{t 1} \dot{\omega}_{t}$ in the pitch equation (28) and $I_{m 1} \dot{\omega}_{m} \cos \psi$ in the yaw equation (29), which, in [2], are considered negligible. However, in contrast to [2], here we have an explicit expression for each term appearing in the equations. In addition, we note that the Coriolis term with the speed of the tail rotor, which appears in [2, eq.1], turns out to be null.

Finally, we point out some omissions in the the Newtonian model of [3] and [4], and in the Lagrangian model of [3]. To start with, we note that the Newtonian model of [3] contains dimensional inconsistences, see e.g. [3, eq. 8]. The issue was fixed in the updated model presented in [4]. Hence, we will refer to the latter. These Newtonian models assume $h=0$. Hence, those terms which vanish with $h$ are not present. However, we note that the pitch equation [4, eq.5] misses the effect of the reaction torque from the tail propeller (corresponding to $C_{R t} \omega_{t}\left|\omega_{t}\right|$ in (28)) and a Coriolis term (corresponding to $I_{m 1} \omega_{m} \dot{\phi} \sin \psi$ in (28)). On the other hand, it contains a so-called gyroscopic torque, depending of the product of the yaw rate with the square of the speed of the main rotor, which does not appear to be justified. Similarly we note that the yaw equation [4, eq.2] misses the effect of the reaction torque from the main propeller and a Coriolis term (corresponding to $I_{c} \dot{\phi} \dot{\psi} \sin (2 \psi)$ in (29)). The Lagrangian model of [3] does not assume $h=0$ and in fact contains those additional terms which vanishes with $h$. However, we note that, similarly to the case of [4], the effects of the reaction torques on pitch and yaw equations are missing, together with some Coriolis terms, while the same gyroscopic torque of [4] is present in the pitch equation which still does not appear to be justified.

\section{Parameters}

In this section we provide numerical values of all parameters. In Table 1 we provide the values of some physical parameters, some of which were measured, while others were obtained from the manufacturer [6, 7]. The TRMS was disassembled in order to measure the weights of each component. The electrical parameters were obtained from the technical specification of the motors' manufacturer [14]. The values of other parameters were either estimated or calculated from the values of Table 1 as described in the following subsections. The reader is then referred to Table 2 for the numerical values of all parameters which appear in the equations of motion (28)-(31). 
Table 1: TRMS parameters

\begin{tabular}{l|c|c}
\hline parameter & value & $\mathrm{unit}$ \\
\hline$l_{m}:$ length of main beam & 0.254 & $\mathrm{~m}$ \\
$l_{t}:$ length of tail beam & 0.275 & $\mathrm{~m}$ \\
$l_{b}:$ length of counter balance beam & 0.250 & $\mathrm{~m}$ \\
$l_{c b}:$ dist. from counter bal. weight to pivot & 0.240 & $\mathrm{~m}$ \\
$r_{s m}:$ radius of main shield & 0.155 & $\mathrm{~m}$ \\
$r_{s t}:$ radius of tail shield & 0.100 & $\mathrm{~m}$ \\
$r_{p m}:$ radius of main propeller & 0.145 & $\mathrm{~m}$ \\
$r_{p t}:$ radius of tail propeller & 0.090 & $\mathrm{~m}$ \\
$h:$ length of pivoted beam & 0.06 & $\mathrm{~m}$ \\
$m_{m}:$ mass of main beam & 0.054 & $\mathrm{~kg}$ \\
$m_{t}:$ mass of tail beam & 0.058 & $\mathrm{~kg}$ \\
$m_{b}:$ mass of counter bal. beam & 0.021 & $\mathrm{~kg}$ \\
$m_{c b}:$ mass of counter bal. weight & 0.068 & $\mathrm{~kg}$ \\
$m_{s m}:$ mass of main shield & 0.159 & $\mathrm{~kg}$ \\
$m_{s t}:$ mass of tail shield & 0.091 & $\mathrm{~kg}$ \\
$m_{p m}:$ mass of main propeller & 0.039 & $\mathrm{~kg}$ \\
$m_{p t}:$ mass of tail propeller & 0.014 & $\mathrm{~kg}$ \\
$m_{m m}:$ mass of main motor and holder & 0.237 & $\mathrm{~kg}$ \\
$m_{m t}:$ mass of tail motor and holder & 0.237 & $\mathrm{~kg}$ \\
$m_{h}:$ mass of pivoted beam & 0.01 & $\mathrm{~kg}$ \\
$R_{m}, R_{t}:$ motors' resistance & 8 & $\Omega$ \\
$L_{m m}, L_{m t}:$ motors' inductance & 0.86 & $\mathrm{mH}$ \\
$k_{v m}, k_{v t}:$ motors' EMF constant & 0.0202 & $\mathrm{~N} \mathrm{~m} \mathrm{~A}-1$ \\
$k_{t m}, k_{t t}:$ motors' torque constant & 0.0202 & $\mathrm{~V} \mathrm{rad}-1$ \\
$k_{u m}:$ main motor's input gain & $\mathrm{No} \mathrm{units}$ \\
$k_{u t}:$ tail motor's input gain & 6.5 & $\mathrm{No} \mathrm{units}$ \\
\hline & & \\
& &
\end{tabular}

\subsection{Propellers' coefficients}

The propellers' thrust and reactive torque coefficients were estimated from experiments. For illustration, the experimental setup for measuring the main propeller's thrust is depicted in Figure 6. In the setup, a weight is attached to the main rotor and placed on an electronic scale, and a counterweight is added to achieve horizontal equilibrium. In addition, the yaw angle is fixed in order to avoid the effect of the reactive torque. The rotors' speeds are obtained from the motors' tachometers. The thrust of the main propeller is then obtained from the measured lifting force as:

$$
T_{m}=F_{L} \frac{\left(l_{m}+r_{s m}\right)}{l_{m}}
$$

The corresponding coefficient can be obtained using a least squares fitting of the experimental curve with a function of the form $C \omega|\omega|$. The other coefficients for thrust and reactive torques were estimated in a similar way. The estimated 


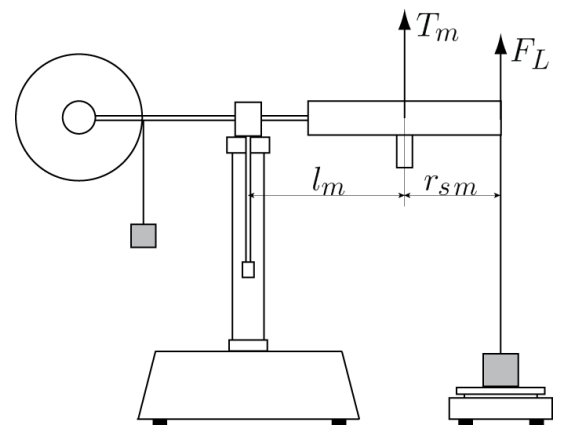

Figure 6: Experimental setup (main propeller thrust)

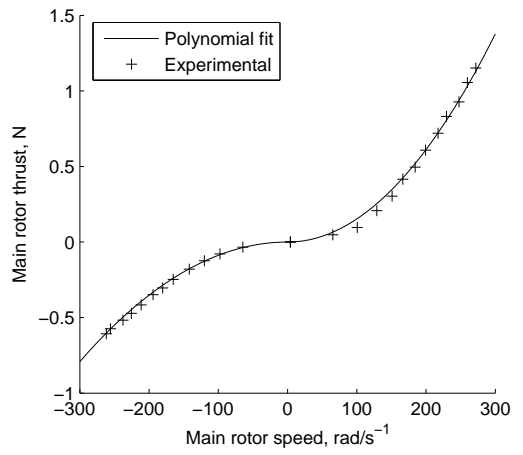

Figure 7: Main propeller static characteristic

values of all coefficients are provided in Table 2, with superscripts + and indicating positive and negative rotor speeds respectively.

\subsection{Calculated parameters}

The inertia parameters can be calculated from the parameters of Table 1 assuming that each component of the TRMS is a simple geometric object.

Each propeller is assumed to be a thin rod. Thus neglecting the motor's rotor inertia we have

$$
\begin{aligned}
& I_{m 1}=\frac{1}{12} m_{p m}\left(2 r_{p m}\right)^{2}, \quad I_{m 2}=0, \quad I_{m 3}=I_{m 1}, \\
& I_{t 1}=\frac{1}{12} m_{p t}\left(2 r_{p t}\right)^{2}, \quad I_{t 2}=0, \quad I_{t 3}=I_{t 1} .
\end{aligned}
$$

The motor's rotor inertia has nominal value $13 \mathrm{gcm}^{2}$ according to the manufacturer's technical specifications, which is indeed negligible with respect to the values calculated above.

The inertia tensor of the TRMS body about the body-fixed reference frame is equal to the sum of inertia tensors of all constituent parts about the same

frame. The main, tail and counterbalance beams are considered as thin rods, the main and tail shields as thin cylindrical shells with open ends, and the motors and counterbalance weight as a point masses. Since the motor's rotors were considered negligible in the rotating parts all the masses of the motors are thus assigned to their static parts. Hence, we assume $M_{m}=m_{p m}$ and $M_{t}=m_{p t}$ in (23). Thus, we have:

$$
\begin{aligned}
I_{b 1} & =\frac{1}{3} m_{m} l_{m}^{2}+\frac{1}{3} m_{t} l_{t}^{2}+\frac{1}{3} m_{b} l_{b}^{2}+\frac{1}{2} m_{s m} r_{s m}^{2}+m_{s m} l_{m}^{2} \\
& +m_{s t} r_{s t}^{2}+m_{s t} l_{t}^{2}+m_{c b} l_{c b}^{2}+m_{m m} l_{m}^{2}+m_{m t} l_{t}^{2} \\
I_{b 2} & =\frac{1}{3} m_{b} l_{b}^{2}+\frac{1}{2} m_{s m} r_{s m}^{2}+\frac{1}{2} m_{s t} r_{s t}^{2}+m_{c b} l_{c b}^{2}
\end{aligned}
$$



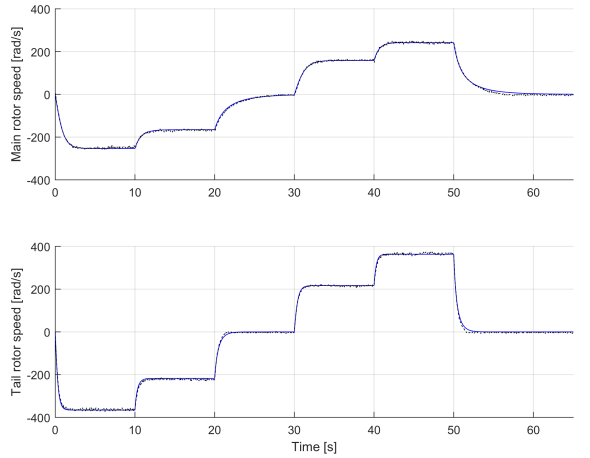

Figure 8: Rotors subsystem identification: identified (solid) and experimental (dashed) responses.
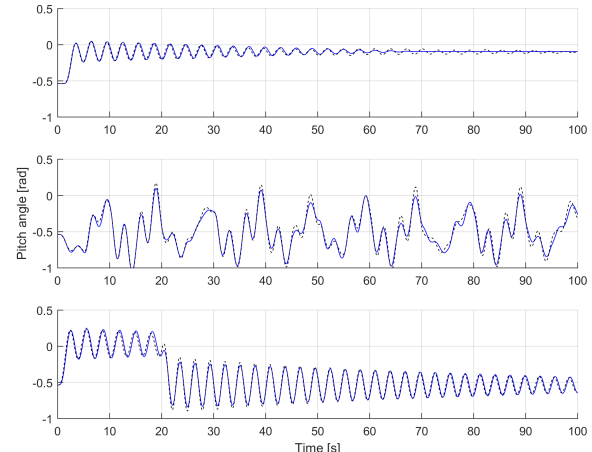

Figure 9: Pitch subsystem identification: identified (solid) and experimental (dashed) responses.

$$
\begin{aligned}
I_{b 3} & =\frac{1}{3} m_{m} l_{m}^{2}+\frac{1}{3} m_{t} l_{t}^{2}+m_{s m} r_{s m}^{2}+m_{s m} l_{m}^{2}+\frac{1}{2} m_{s t} r_{s t}^{2}+m_{s t} l_{t}^{2} \\
& +m_{m m} l_{m}^{2}+m_{m t} l_{t}^{2}
\end{aligned}
$$

The pivoted beam is assumed to be a thin rod of length $h$ and mass $m_{h}$. Hence we obtain:

$$
I_{p}=\frac{1}{3} m_{h} h^{2}
$$

The total masses of the TRMS body and of the aggregate body are respectively given by

$$
\begin{aligned}
& M_{b}=m_{m}+m_{t}+m_{b}+m_{s m}+m_{s t}+m_{c b}+m_{m m}+m_{m t}, \\
& M_{a}=M_{b}+m_{p m}+m_{p t} .
\end{aligned}
$$

Finally, the coordinates of the centres of mass of the TRMS body and of the aggregate body are determined respectively as

$$
\begin{aligned}
& y_{G b}= \frac{1}{M_{b}}\left(\frac{1}{2} l_{m} m_{m}-\frac{1}{2} l_{t} m_{t}+l_{m} m_{s m}-l_{t} m_{s t}+l_{m} m_{m m}-l_{t} m_{m t}\right), \\
& z_{G b}=\frac{1}{M_{b}}\left(-\frac{1}{2} l_{b} m_{b}-l_{c b} m_{c b}\right),\left.+l_{m} m_{p m}-l_{t} m_{p t}\right), \\
& y_{G a}=\frac{1}{M_{a}}\left(\frac{1}{2} l_{m} m_{m}-\frac{1}{2} l_{t} m_{t}+l_{m} m_{s m}-l_{t} m_{s t}+l_{m} m_{m m}-l_{t} m_{m t}\right. \\
& z_{G a}=\frac{1}{M_{a}}\left(-\frac{1}{2} l_{b} m_{b}-l_{c b} m_{c b}\right) .
\end{aligned}
$$

The numerical values of all calculated parameters are provided in Table 2. 
Table 2: Parameters of the equations of motion (28)-(31)

\begin{tabular}{|c|c|c|c|c|}
\hline parameter & estimated & calculated & identified & unit \\
\hline$I_{m 1}$ & - & $2.7332 \times 10^{-4}$ & $1.7249 \times 10^{-4}$ & $\mathrm{~kg} \mathrm{~m}^{2}$ \\
\hline$I_{t 1}$ & - & $3.7800 \times 10^{-5}$ & $3.2170 \times 10^{-5}$ & $\mathrm{~kg} \mathrm{~m}^{2}$ \\
\hline$I_{\psi}$ & - & $6.3764 \times 10^{-2}$ & $6.1644 \times 10^{-2}$ & $\mathrm{~kg} \mathrm{~m}^{2}$ \\
\hline$I_{\phi}$ & - & $1.0408 \times 10^{-2}$ & $2.1117 \times 10^{-2}$ & $\mathrm{~kg} \mathrm{~m}^{2}$ \\
\hline$I_{c}$ & - & $5.4244 \times 10^{-2}$ & $8.3286 \times 10^{-2}$ & $\mathrm{~kg} \mathrm{~m}^{2}$ \\
\hline$f_{v \psi}$ & - & - & $4.2150 \times 10^{-4}$ & $\mathrm{Nm} \mathrm{rad}^{-1} \mathrm{~s}$ \\
\hline$f_{c \psi}$ & - & - & $4.0548 \times 10^{-4}$ & $\mathrm{Nm}$ \\
\hline$f_{v \phi}$ & - & - & $1.1243 \times 10^{-4}$ & $\mathrm{Nm} \mathrm{rad}^{-1} \mathrm{~s}$ \\
\hline$f_{c \phi}$ & - & - & $1.1758 \times 10^{-5}$ & $\mathrm{Nm}$ \\
\hline$f_{v m}$ & - & - & $4.1993 \times 10^{-6}$ & $\mathrm{Nm} \mathrm{rad}^{-1} \mathrm{~s}$ \\
\hline$f_{v t}$ & - & - & $3.2498 \times 10^{-6}$ & $\mathrm{Nm} \mathrm{rad}^{-1} \mathrm{~s}$ \\
\hline$C_{T m}^{+}$ & $1.53 \times 10^{-5}$ & - & $1.4971 \times 10^{-5}$ & $\mathrm{Ns}^{2} \mathrm{rad}^{-2}$ \\
\hline$C_{T m}^{--m}$ & $8.8 \times 10^{-6}$ & - & $8.9974 \times 10^{-6}$ & $\mathrm{Ns}^{2} \mathrm{rad}^{-2}$ \\
\hline$C_{T t}^{+}$ & $3.25 \times 10^{-6}$ & - & $3.7755 \times 10^{-6}$ & $\mathrm{Ns}^{2} \mathrm{rad}^{-2}$ \\
\hline$C_{T t}^{-}$ & $1.72 \times 10^{-6}$ & - & $2.3046 \times 10^{-6}$ & $\mathrm{~N} \mathrm{~s}^{2} \mathrm{rad}^{-2}$ \\
\hline$C_{R m}^{+}$ & $4.9 \times 10^{-7}$ & - & $5.0582 \times 10^{-7}$ & $\mathrm{Nm} \mathrm{s}^{2} \mathrm{rad}^{-2}$ \\
\hline$C_{R m}^{-}$ & $4.1 \times 10^{-7}$ & - & $4.5047 \times 10^{-7}$ & $\mathrm{Nm} \mathrm{s}^{2} \mathrm{rad}^{-2}$ \\
\hline$C_{R t}^{+m}$ & $9.70 \times 10^{-8}$ & - & $9.7031 \times 10^{-8}$ & $\mathrm{Nm} \mathrm{s}^{2} \mathrm{rad}^{-2}$ \\
\hline$C_{R t}^{-c}$ & $9.70 \times 10^{-8}$ & - & $9.9176 \times 10^{-8}$ & $\mathrm{Nms}^{2} \mathrm{rad}^{-2}$ \\
\hline$C_{c}$ & - & - & $9.8664 \times 10^{-3}$ & $\mathrm{Nm} \mathrm{mad}^{-1}$ \\
\hline$\psi_{0}$ & - & -0.6801 & -0.5369 & $\mathrm{rad}$ \\
\hline$\phi_{0}$ & - & - & 0.2500 & rad \\
\hline$G_{z}$ & - & -0.18585 & -0.25254 & $\mathrm{Nm}$ \\
\hline$G_{y}$ & - & 0.15032 & - & $\mathrm{Nm}$ \\
\hline$H_{z}$ & - & $-1.1367 \times 10^{-3}$ & $-1.5446 \times 10^{-3}$ & $\mathrm{~kg} \mathrm{~m}^{2}$ \\
\hline$H_{y}$ & - & $6.6120 \times 10^{-3}$ & - & $\mathrm{kg} \mathrm{m}^{2}$ \\
\hline
\end{tabular}

\subsection{Identification of parameters}

The estimated and calculated parameters were adjusted further, and the remaining parameters (friction and cable coefficients) were identified, using experimental responses and an output-error least-squares criterion. Identification data were sampled with sampling time $T_{c}=0.1 \mathrm{~s}$. The identification criterion was optimized over positive values of the parameters using the constrained local optimization algorithms of Matlab starting from the parameters' nominal values, which were taken as null if not available. All identified parameters' values are reported in Table 2 .

The identification was carried out separately for the following four subsystems, the main rotor, tail rotor, pitch angle and the yaw angle:

- In the rotors' subsystems, the nominal electrical parameters were assumed to be correct. The moment of inertia $I_{m 1}$, the reaction torque coefficients $C_{R m}^{+/-}$ and the friction coefficient $f_{v m}$ in equation (30), and the moment of inertia $I_{t 1}$, the reaction torque coefficients $C_{R t}^{+/-}$and the friction coefficient $f_{v t}$ in equation 


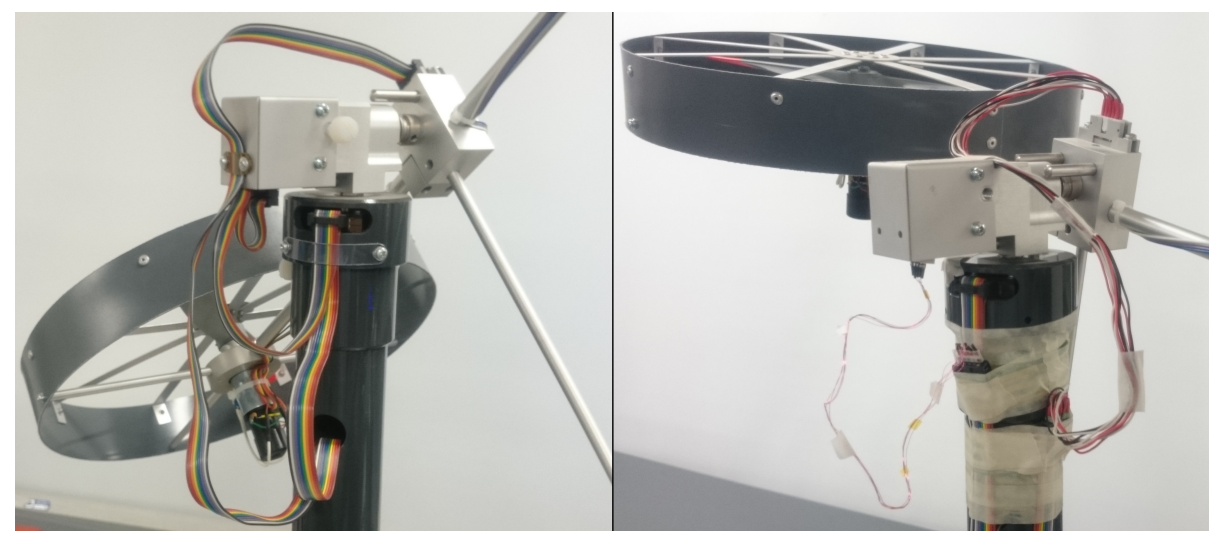

Figure 10: original TRMS wiring with flat cables (left) and new wiring (right).

(31), were identified from velocity step responses obtained from isolated rotors' experiments. The data were read directly from the motors' tachometers. The experiments are illustrated in Figure 8.

- The pitch angle subsystem was treated in $1 \mathrm{DOF}$ mode, i.e. with fixed yaw angle, in order to eliminate the effects of cross-couplings. Hence, in this case, the model was determined by equations (28) and (30) with $\ddot{\phi}=\dot{\phi}=0$ and the rotors' parameters identified above. In the rest position, the pitch angle $\psi_{0}$, which is determined solely by the centre of mass of the aggregate body, was measured to be sightly different from the value predicted by equations (42-43). In fact, the centre of mass of the main motor lies slightly below the $y$-axis, while in equation (43) we assumed it lying on the $y$-axis. From equation (28), taking all velocities equal to zero, we obtain the static equation $G_{z} \sin \psi_{0}-G_{y} \cos \psi_{0}=0$ which yields an adjusted value of $G_{z}$. Using equations (41) and (43), we also obtained a corresponding adjusted value of $H_{z}$. Then, the parameters $I_{\psi}, f_{v \psi}$, $f_{c \psi}$ and $C_{T m}^{+/-}$, were identified from pitch responses obtained with the following three main motor's inputs: a step input with amplitude 1.25 , a square wave input with amplitudes $[-1,1]$ at $1 \mathrm{~Hz}$ and a square input with amplitude 1.5 and width 20s. Note that the last part of the third experiment is the free response of the system. The three experiments are illustrated in Figure 9.

- The parameters of the yaw angle subsystem were identified from experiments carried out in the standard 2DOF mode. In its original configuration, the TRMS setup includes two flat cables which connect sensors and motors to the base. In preliminary experiments, it was noted that these cables exhibit a complex dynamic behaviour, depending on the changes of their position and shape during the experiment, which appears difficult to capture using models derived from first principles and impinges the repeatability of experiments. Hence, with the purpose of removing un-modelled dynamics as much as possible, the TRMS setup was re-wired using lighter wires to connect the motors (26 AWG $0.22 \mathrm{~mm}^{2}$ servo wire) and microwires to connect the sensors (38 AWG $0.018 \mathrm{~mm}^{2}$ solderable enamelled copper wire), see Figure 10. 
The new wiring appears to generate a residual small torque which, possibly together with other un-modelled forces, determines the rest angle $\phi_{0}$ reported in Table 2. Without the action of the flat cables, the dynamics of the yaw is virtually unstable and the beam hits the limits of the yaw range in almost any experiment. Hence, identification experiments were con-

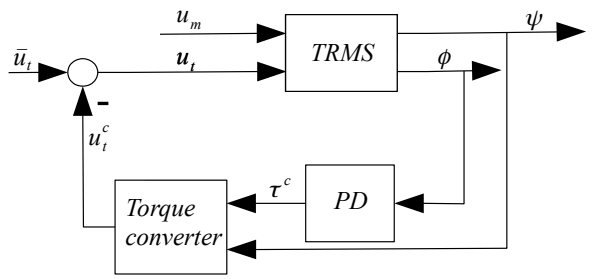

Figure 11: Yaw identification experiment ducted with a feedback controller stabilising the yaw around $\phi=0$. The yaw feedback control generated a control torque along the yaw angle according to a Proportional Derivative (PD) action converted into a tail motor control signal (see Figure 11). The reader is referred to the next section for details on the implementation of the yaw stabilising controller. In the identification experiments, the system was excited with inputs $u_{m}$ and $\bar{u}_{t}$ as depicted in Figure 11. In the identification criterion, the signals $u_{m}$ and $\bar{u}_{t}$ were taken as inputs and $\psi$ and $\phi$ as outputs. Hence, the feedback controller was implemented also in the model. This was required because, as mentioned, the open-loop yaw dynamics is almost unstable. In the standard 2DOF mode, the full equations of motion (28)-(31) were implemented in a Simulink model compiled in rapid accelerator mode with tunable parameters and fixed step-size $T_{s}=0.001 \mathrm{~s}$. In the model, the time-varying inertia matrix represented by the left-hand sides of equations (28)-(31) was implemented via symbolic matrix inversion. The three identification experiments used to identify parameters $I_{\phi}, I_{c}, f_{v \phi}, f_{c \phi}, C_{T t}^{+/-}$and $C_{c}$ are illustrated in Section 5.

\section{Implementation of the yaw stabilising controller}

In this section, we describe the implementation of the yaw stabilising control loop used in the identification and validation experiments (Figure 11). The PD controller was implemented in discrete-time with sampling time $T_{s}=0.001$. It can be verified with simple preliminary experiments that the open-loop cut-off frequency of the TRMS is below $1 \mathrm{~Hz}$. Hence, $T_{s}$ is a suitable sampling time. The derivative action was implemented in discrete-time using a "textbook" backward difference discretization scheme, see e.g. [15]. The resulting PD controller in discrete-time form is described by:

$$
P D(z)=K_{p}+\frac{K_{d} N}{1+N T_{s}} \frac{z-1}{z-\frac{1}{1+N T_{s}}}
$$

with $N=20$. The output $\tau^{c}$ of the PD controller is interpreted to be a control torque to be applied along the yaw angle. In the implementation, the control torque needs to be converted to the actual control signal for the tail motor. In order to do this, the control torque $\tau^{c}$ is first converted into the corresponding 
tail rotor's speed:

$$
\omega_{t}^{c}=\frac{\operatorname{sgn}\left(\tau^{c}\right)}{\sqrt{C_{T t}^{+/-}}} \sqrt{\frac{\left|\tau^{c}\right|}{l_{t} \cos \psi}} .
$$

Then the control input $u_{c}$ can be obtained from equations (3) and (31). In equation (31) the term with the yaw angle acceleration can be omitted assuming that its magnitude is negligibly smaller than the rotor's acceleration. Further, making a steady state approximation, i.e. taking the tail rotor's acceleration equal to zero, the corresponding control input is given by:

$$
u_{t}^{c}=\frac{1}{k_{u t}}\left(\frac{R_{t} f_{v t}}{k_{t t}}+k_{v t}\right) \omega_{t}^{c}+\frac{R_{t} C_{R t}^{+/-}}{k_{u t} k_{t t}} \omega_{t}^{c}\left|\omega_{t}^{c}\right| .
$$

Note that the expression in (44) depends on the parameter $C_{T t}^{+/-}$. Hence, in the identification experiments, the estimated values of this parameter, from the first column of Table 2, were used to implement the controller. The controller was tuned by trial-and-error, during preliminary experiments, firstly by implementing the proportional action and then by increasing the derivative action to achieve stability. The so-obtained control gains were $K_{p}=0.1$ and $K_{d}=0.1$. The resulting open-loop dynamics between $\bar{u}_{t}$ and $\phi$ maintains a cut-off frequency below $1 \mathrm{~Hz}$.

\section{Experimental validation}

In this section, we illustrate the performance of the full 2DOF TRMS model by comparing the simulated responses obtained from the model with the experimental responses obtained from the real TRMS setup. In each experiment, the same inputs $u_{m}$ and $\bar{u}_{t}$ were applied independently to the TRMS model and to the real TRMS setup and the simulated and real $\psi$ and $\phi$ outputs were compared. Both simulated and experimental responses were obtained using the stabilizing controller of the yaw dynamics illustrated in Figure 11. The experiments presented are separated in two groups: identification experiments were used to identify the parameters of the yaw subsystem as discussed in Section 3.3, validation experiments were used to assess the generalisation performance of the model for inputs not used in the identification experiments. The responses obtained in the identification experiments are displayed in Figures 12-14 and the responses obtained in the validation experiments are displayed in Figures 15-17. The figures' captions provide the details of the inputs used in each experiment. In Table 3 we provide quantitative measures of the performance of the model in each experiment. In the first two columns of the table we report the mean squared errors (mse) between the real and simulated pitch and yaw responses, which is an absolute measure of the energy of the modeling errors. In the third and fourth columns we report the ratio between the mse values of the pitch and yaw responses and the mean squared values of $\psi-\psi_{0}$ and of $\phi-\phi_{0}$ respectively. This is a relative measure of the energy of the modeling errors with respect to 


\begin{tabular}{c|l|c|c|c|c|c|}
\hline & Fig. & $\mathrm{mse}_{\psi}$ & $\mathrm{mse}_{\phi}$ & $\mathrm{rmse}_{\psi}$ & $\mathrm{rmse}_{\phi}$ & $\mathrm{rmse}_{\psi \phi}$ \\
\hline \multirow{2}{*}{$\begin{array}{c}\text { Identification } \\
\text { experiments }\end{array}$} & 12 & $3.63 \times 10^{-3}$ & $1.50 \times 10^{-3}$ & 0.040 & 0.014 & 0.026 \\
\cline { 2 - 7 } & 13 & $8.42 \times 10^{-4}$ & $1.48 \times 10^{-3}$ & 0.073 & 0.008 & 0.012 \\
\cline { 2 - 7 } & 14 & $3.17 \times 10^{-3}$ & $3.21 \times 10^{-3}$ & 0.027 & 0.011 & 0.016 \\
\hline \multirow{3}{*}{$\begin{array}{c}\text { Validation } \\
\text { experiments }\end{array}$} & 15 & $2.55 \times 10^{-3}$ & $1.03 \times 10^{-3}$ & 0.026 & 0.011 & 0.019 \\
\cline { 2 - 7 } & 16 & $5.98 \times 10^{-4}$ & $1.47 \times 10^{-3}$ & 0.183 & 0.007 & 0.010 \\
\cline { 2 - 7 } & 17 & $7.90 \times 10^{-3}$ & $2.12 \times 10^{-3}$ & 0.077 & 0.016 & 0.042 \\
\cline { 2 - 7 } & 18 & $1.02 \times 10^{-3}$ & $5.37 \times 10^{-4}$ & 0.038 & 0.009 & 0.018 \\
\hline
\end{tabular}

Table 3: mean square errors

the energy of the outputs, which can be used to compare the performance of the model in different experiments. In the fifth column, we report the ratio between the sum of the mse values of the pitch and yaw responses and the sum of the mean squared values of $\psi-\psi_{0}$ and of $\phi-\phi_{0}$. This is again a relative measure of the energy of the modeling errors with respect to the energy of the outputs but considering the pitch and yaw responses together as a two-dimensional signal. It is quite straightforward to see that the model has a very good performance by inspecting the values of the absolute and relative errors. It can be seen that the worst case pitch relative mse value is $18.3 \%$, obtained in the validation experiment of Figure 16 (which is the experiment with the lowest pitch output energy), that the worst case yaw relative mse value is $1.6 \%$, obtained in the validation experiment of Figure 17, and that the worst case two-dimensional relative mse value is $4.2 \%$ obtained in the validation experiment of Figure 17. Most importantly, it can be seen that the absolute and relative mse values remain at similar levels in the identification and validation experiments thus indicating a very good generalization performance of the model. In [3], the absolute mse values of similar experiments were reported. However, the comparison with the results of [3] can only be indicative. This is because only two cumulative mse values for the pitch and yaw responses covering several experiments of different lengths were reported in [3]. Under these premises, we note that the mse values of the first two columns of Table 3 are consistently smaller than the mse values reported in [3, Table 4]. Relative mse values, as those provided in the third to fifth columns of Table 3, have not been reported by previous authors. 

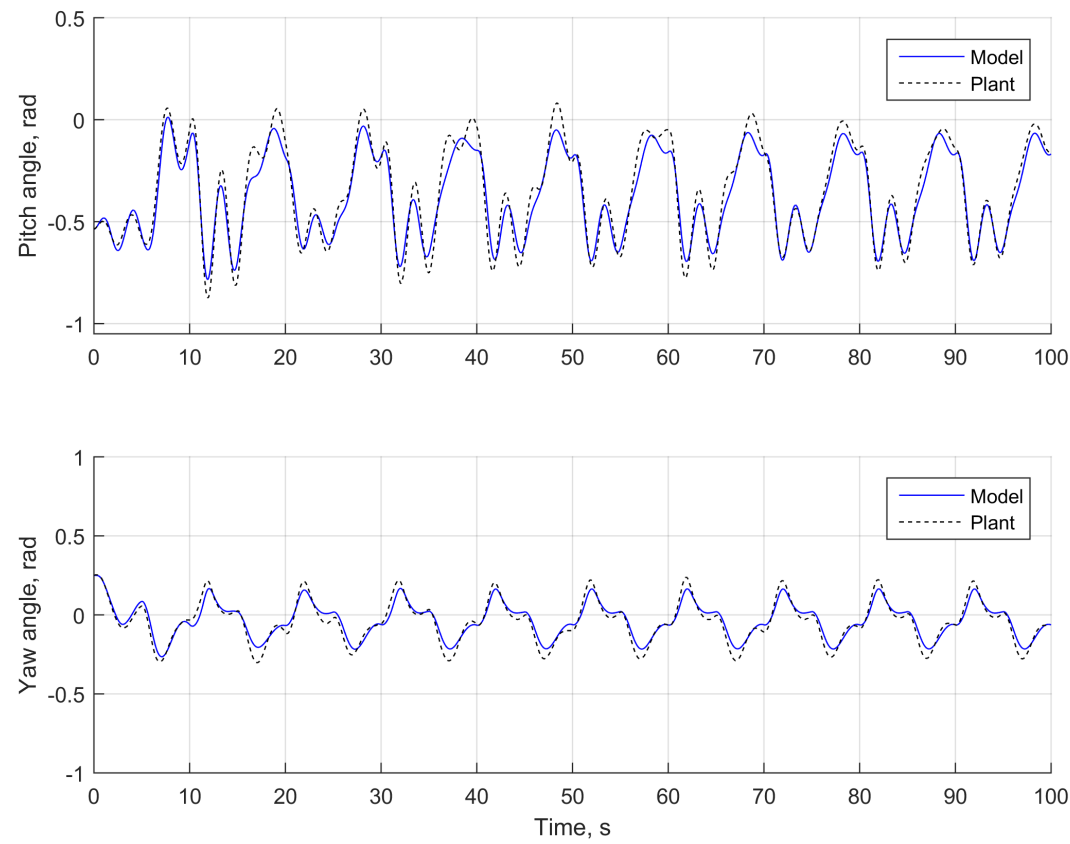

Figure 12: identification experiment, $u_{m}=$ square wave $\{-0.2,1.2\}$ at $0.1 \mathrm{~Hz}$ and $\bar{u}_{t}=0$.
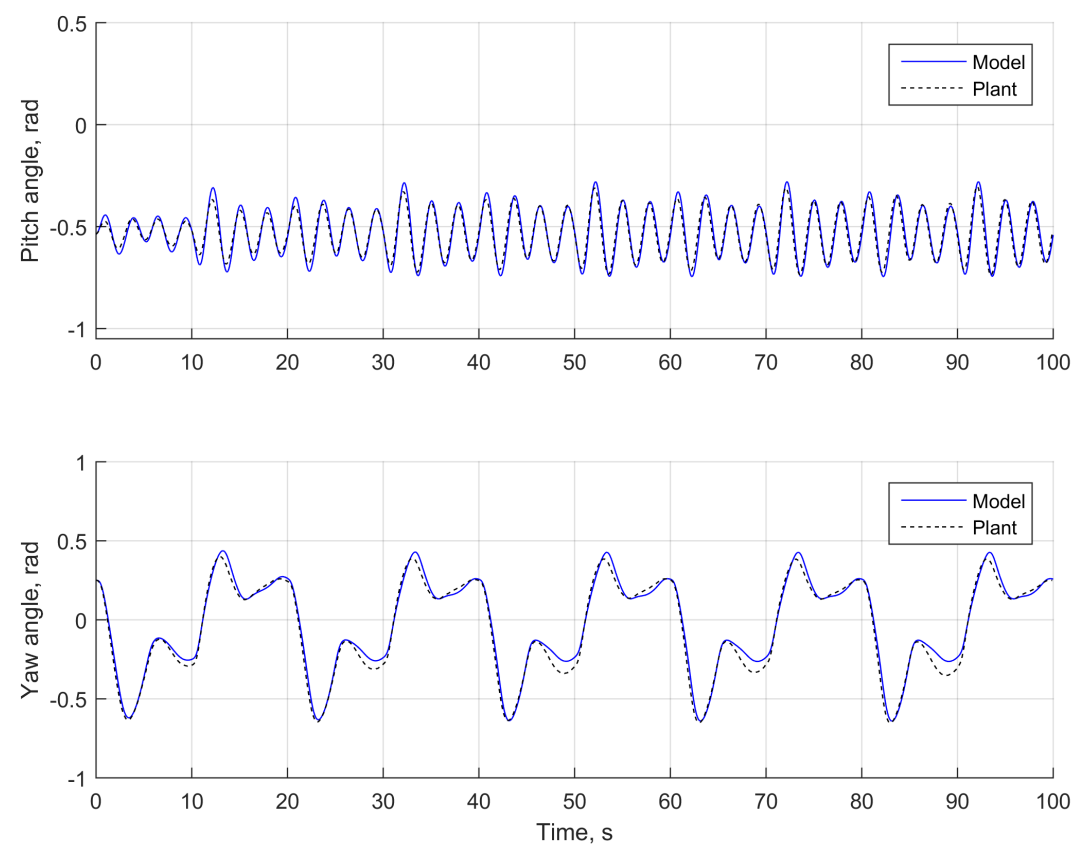

Figure 13: identification experiment, $u_{m}=0$ and $\bar{u}_{t}=$ square wave $\{-1,1\}$ at $0.05 \mathrm{~Hz}$. 

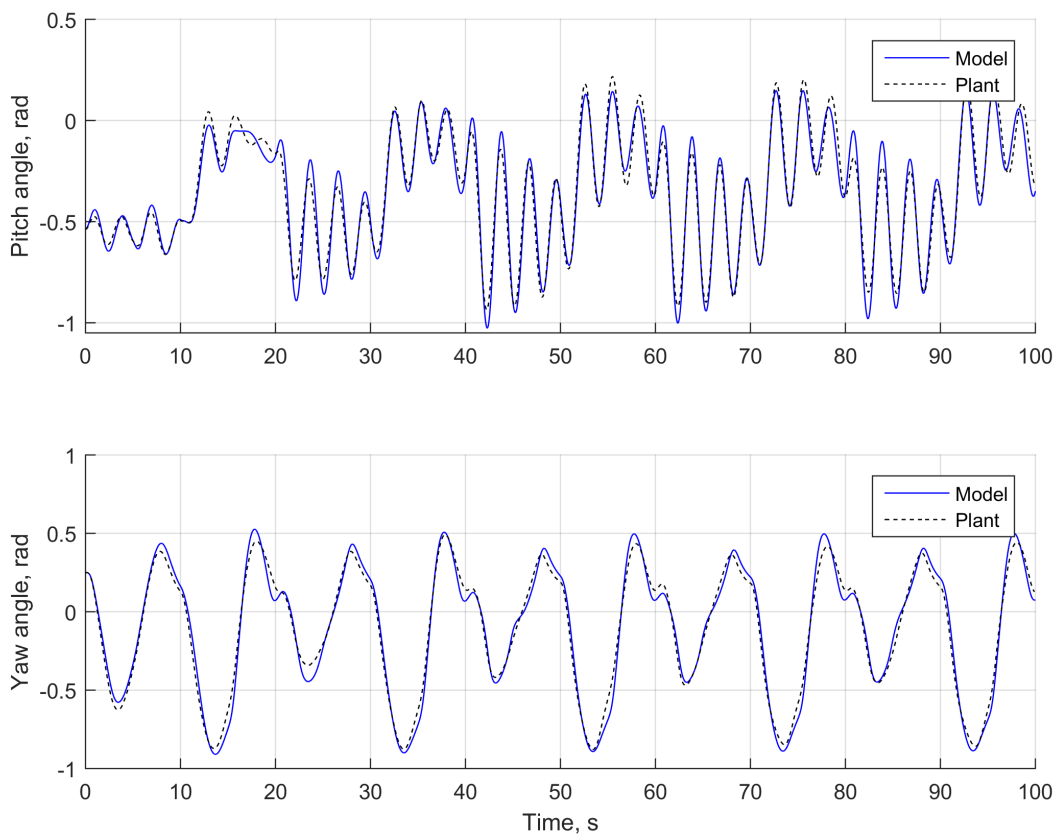

Figure 14: identification experiment, $u_{m}=$ square wave $\{-0.2,1.2\}$ at $0.05 \mathrm{~Hz}$ and $\bar{u}_{t}=$ square wave $\{-1,1\}$ at $0.1 \mathrm{~Hz}$.
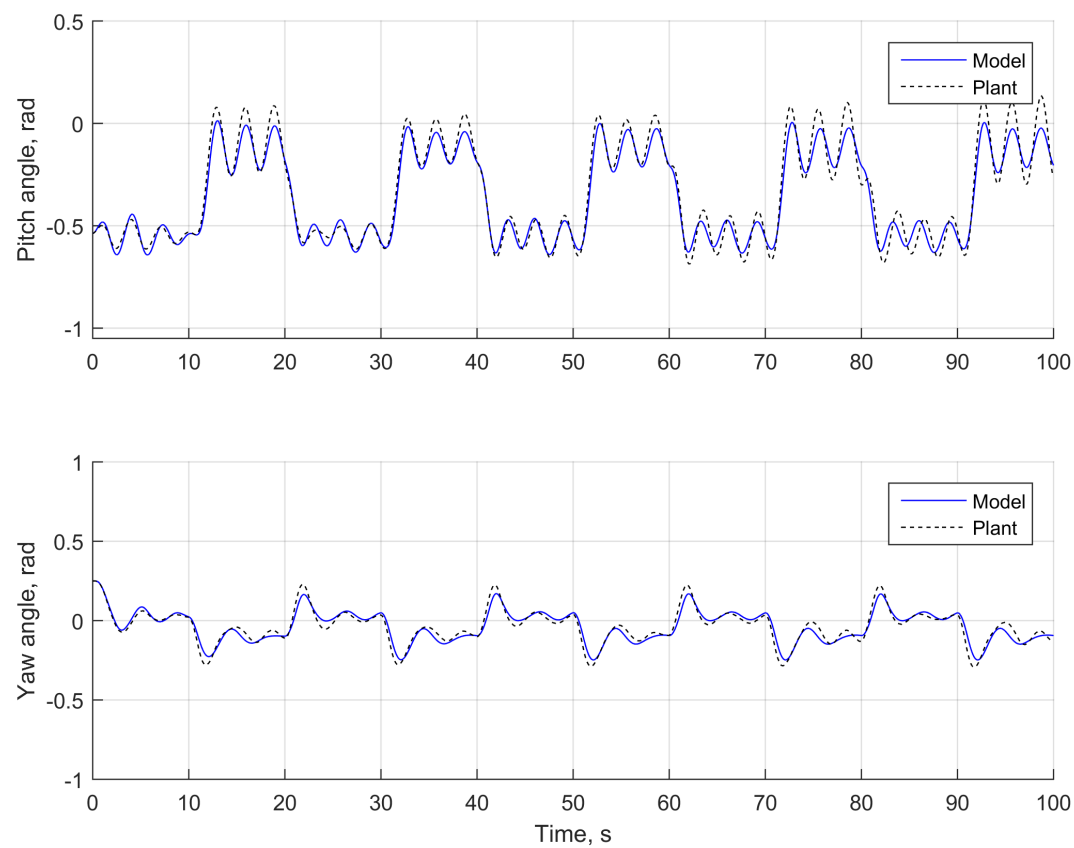

Figure 15: validation experiment, $u_{m}=$ square wave $\{-0.2,1.2\}$ at $0.05 \mathrm{~Hz}$ and $\bar{u}_{t}=0$. 

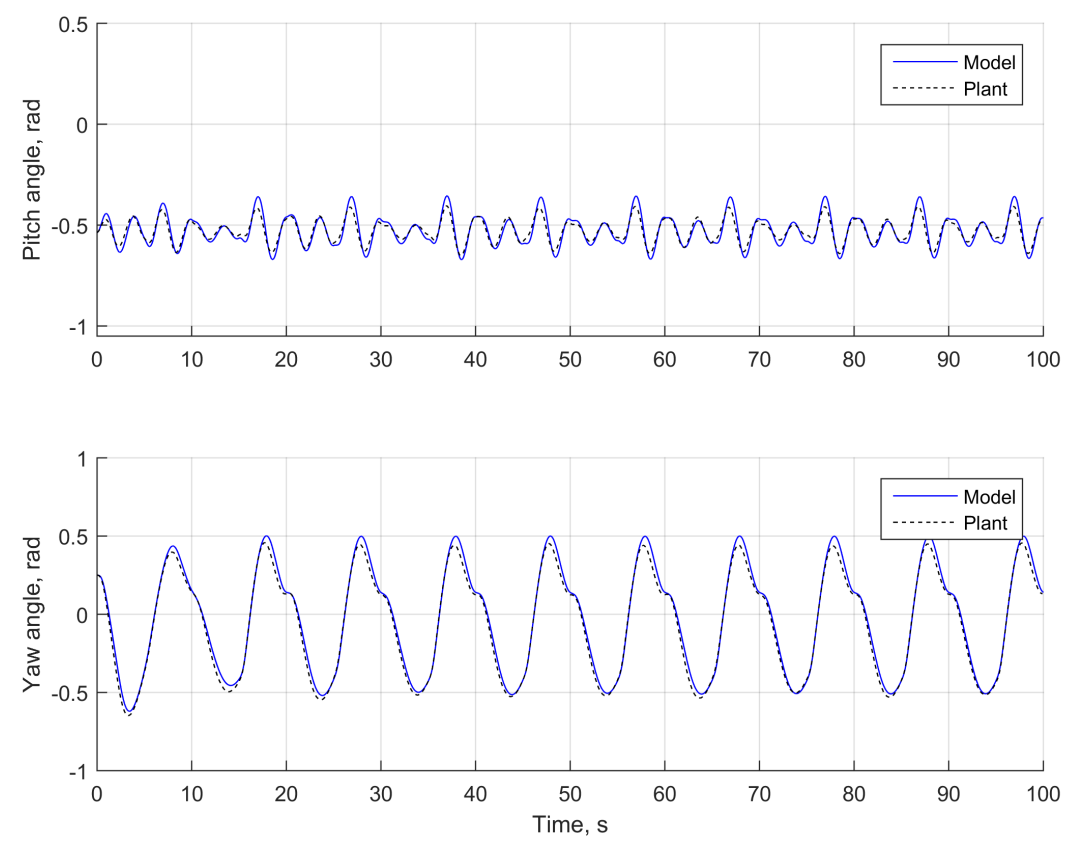

Figure 16: validation experiment, $u_{m}=0$ and $\bar{u}_{t}=$ square wave $\{-1,1\}$ at $0.1 \mathrm{~Hz}$.
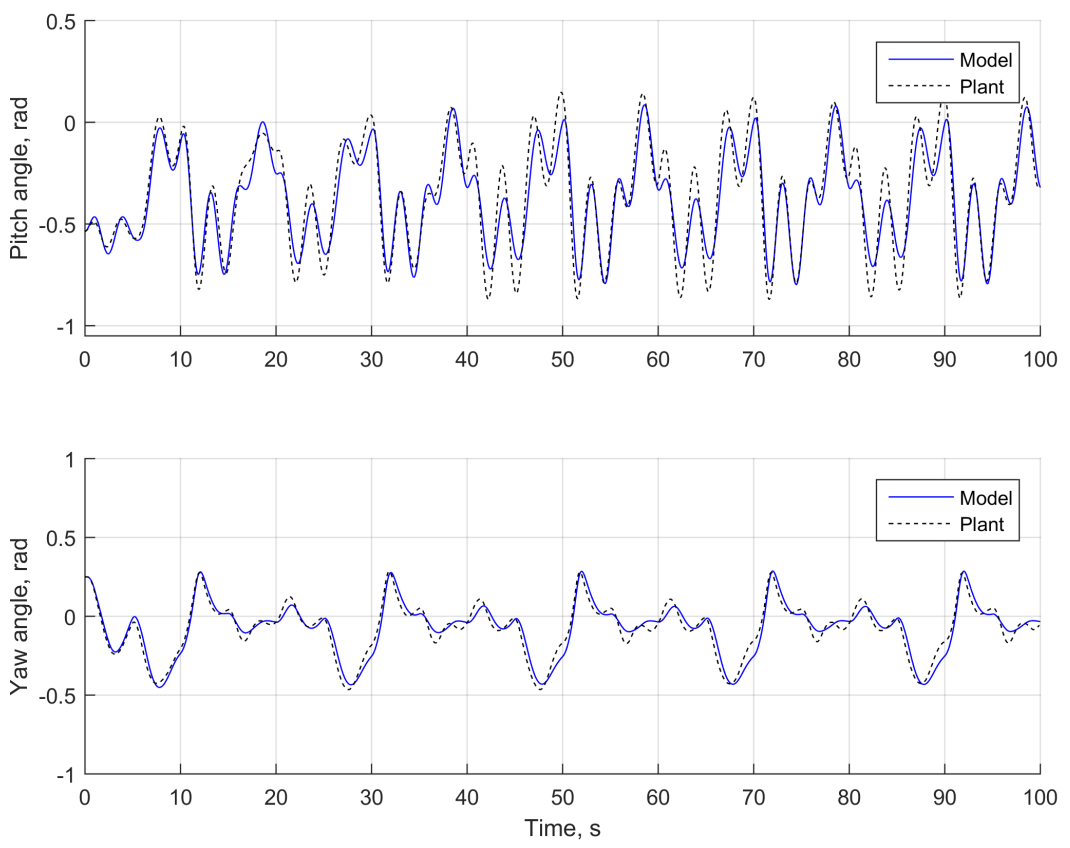

Figure 17: validation experiment, $u_{m}=$ square wave $\{-0.2,1.2\}$ at $0.1 \mathrm{~Hz}$ and $\bar{u}_{t}=$ square wave $\{-0.4,0.2\}$ at $0.05 \mathrm{~Hz}$. 

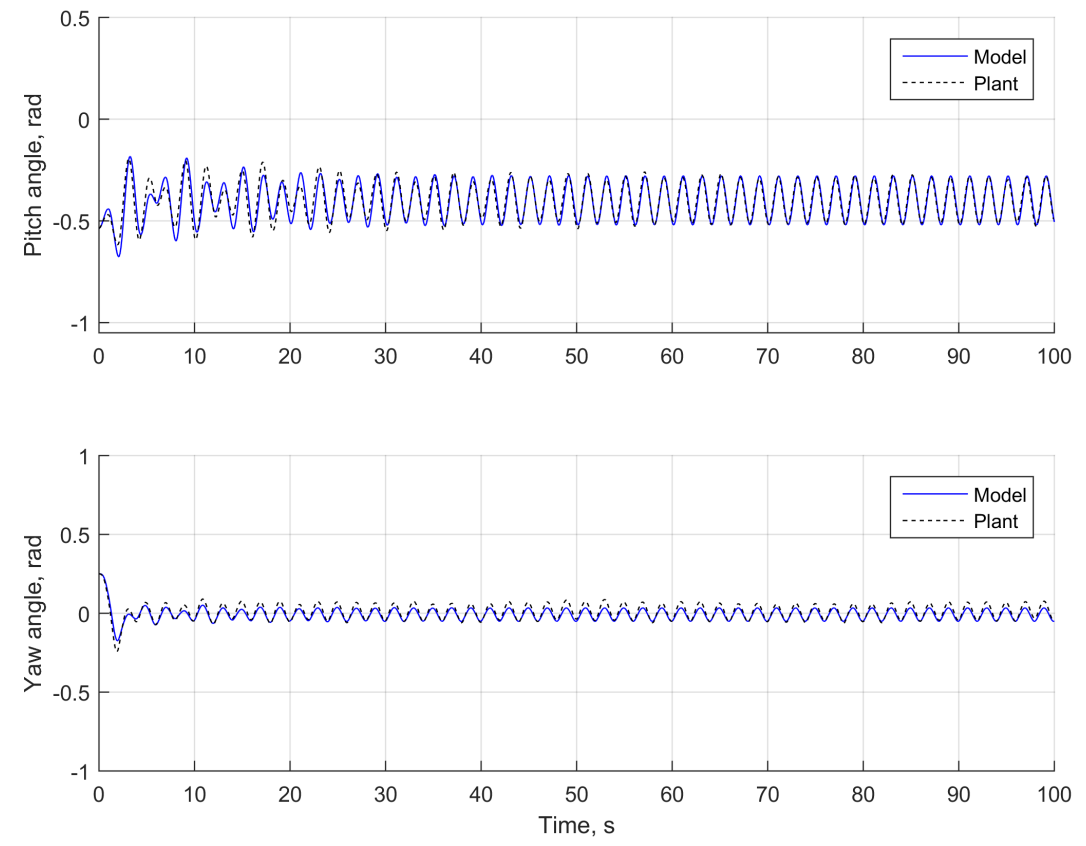

Figure 18: validation experiment, $u_{m}=$ square wave $\{-0.2,1.2\}$ at $0.5 \mathrm{~Hz}$ and $\bar{u}_{t}=$ square wave $\{-1,1\}$ at $0.5 \mathrm{~Hz}$.

\section{Conclusions}

In this contribution, we have rigorously developed a complete dynamic model of the TRMS. The model was identified and validated with experimental data and measures of the performance of the model were reported. We have pointed out some inaccuracies in the derivations of previously reported models of the TRMS. In this work, in order to obtain a fair validation of the model, we have removed the original flat cable of the TRMS set-up. However, for the purpose of robust control design, the original flat cable should be kept and assumed to be un-modelled dynamics. In current work, we are investigating on the implementation of the robust control law developed in $[1,2,16]$, and in particular on the inclusion of an anti-windup scheme to compensate for input saturations $[17,18]$. In addition, we plan to adopt the TRMS as benchmark to develop the use of data-based control tuning methods [19, 20,21].

\section{Acknowledgement}

The authors gratefully acknowledge Mr Rashmikant Patel and Mr Ian Bromley for providing technical assistance in the control lab, Maxon Motor for providing the technical specifications of the TRMS motors, and an anonymous reviewer whose insightful technical comments contributed to improve the paper. 


\section{References}

[1] M. G. Ortega, M. Vargas, C. Vivas, and F.R. Rubio. Robustness improvement of a nonlinear $H_{\infty}$ controller for robot manipulators via saturation functions. Journal of Robotic Systems, 22(8):421-437, 2005.

[2] M. Lopez-Martinez, M.G. Ortega, C. Vivas, and F.R. Rubio. Nonlinear control of a laboratory helicopter with variable speed rotors. Automatica, 43(4):655 - 661, 2007.

[3] A Rahideh and M. H. Shaheed. Mathematical dynamic modelling of a twin-rotor multiple input-multiple output system. Proceedings of the Institution of Mechanical Engineers, Part I: Journal of Systems and Control Engineering, 221(1):89-101, 2007.

[4] D. Rotondo, F. Nejjari, and V. Puig. Quasi-LPV modeling, identification and control of a twin rotor MIMO system. Control Engineering Practice, 21(6):829-846, 2013.

[5] L.M. Belmonte, R. Morales, A. Fernández-Caballero, and J.A. Somolinos. Robust decentralized nonlinear control for a twin rotor mimo system. Sensors, 16(8), 2016.

[6] Feedback Instruments Ltd. Twin Rotor Mimo System. Advanced Teaching Manual 1. 33-007-4M5, 1998.

[7] Feedback Instruments Ltd. Twin Rotor MIMO System Control Experiments. 33-949S, 2006.

[8] P. Mullhaupt, B. Srinivasan, J. Lévine, and D. Bonvin. Control of the toycopter using a flat approximation. IEEE Transactions on Control Systems Technology, 16(5):882-896, 2008.

[9] A. Tastemirov, A. Lecchini-Visintini, and R.M. Morales. Improved model of the twin rotor multiple-input multiple-output system with experimental validation. In Proceedings of the 39th European Rotorcraft Forum, Moscow, 2013.

[10] A.J. Brizard. An introduction to Lagrangian mechanics. World Scientific, 2008.

[11] N. Woodhouse. Introduction to Analytical Dynamics. Springer Undergraduate Mathematics Series. Springer, 2009.

[12] E.L. Houghton and P.W. Carpenter. Aerodynamics for Engineering Students. Butterworth-Heinemann, 2003.

[13] R. Kelly, J. Llamas, and R. Campa. A measurement procedure for viscous and coulomb friction. Instrumentation and Measurement, IEEE Transactions on, 49(4):857 -861, aug 2000. 
[14] Maxon Motor. Technical specifications: DC motor Amax-26 (assembly 202863).

[15] K.J. Astrom and B. Wittenmark. Computer-controlled Systems (3rd Ed.). Prentice-Hall, Inc., 1997.

[16] W Feng and I Postlethwaite. Robust non-linear $H_{\infty}$ adaptive control of robot manipulator motion. Proceedings of the Institution of Mechanical Engineers, Part I: Journal of Systems and Control Engineering, 208(4):221230, 1994.

[17] M.C. Turner, G. Herrmann, and I. Postlethwaite. Incorporating robustness requirements into antiwindup design. IEEE Transactions on Automatic Control, 52(10):1842-1855, 2007.

[18] R.M. Morales, G. Li, and W.P. Heath. Anti-windup and the preservation of robustness against structured norm-bounded uncertainty. International Journal of Robust and Nonlinear Control, 24(17):2640-2652, 2014.

[19] R. Hildebrand, A. Lecchini, G. Solari, and M. Gevers. Asymptotic accuracy of iterative feedback tuning. IEEE Transactions on Automatic Control, 50(8):1182-1185, 2005.

[20] D. Rupp and L. Guzzella. Iterative tuning of internal model controllers with application to air/fuel ratio control. IEEE Transactions on Control Systems Technology, 18(1):177-184, 2010.

[21] M. F. Heertjes, B. Van der Velden, and T. Oomen. Constrained iterative feedback tuning for robust control of a wafer stage system. IEEE Transactions on Control Systems Technology, 24(1):56-66, 2016. 